\title{
Uso do BBC micro: bit como acelerômetro em atividades experimentais para o ensino de física
}

Use of BBC micro: bit as accelerometer in experimental activities for teaching physics

\author{
Diego Santos de Jesus $^{*}{ }^{\circledR}$, João Paulo Casaro Erthal ${ }^{1}$, Roberto Colistete Júnior ${ }^{1}$ \\ ${ }^{1}$ Universidade Federal do Espírito Santo, Vitória, ES, Brasil.
}

\begin{abstract}
Recebido em 30 de junho de 2021. Revisado em 30 de setembro de 2021. Aceito em 11 de novembro de 2021.
Este trabalho investigou a viabilidade de utilização do acelerômetro interno do BBC micro: bit em atividades experimentais. Dois experimentos foram utilizados para testes, sendo que esses tratavam da Segunda Lei de Newton e do movimento de Rotação. Em ambos o objetivo foi medir de forma direta a aceleração e com isso analisar os dados através de estatística descritiva e análises gráficas, gerando resultados que servirão de referencial para alunos em laboratório, além de esclarecer conceitos de difícil visualização e compreensão. Os resultados obtidos auxiliam a compreender melhor o que realmente ocorre nos movimentos de aceleração e podem auxiliar na reorganização de atividades experimentais para o ensino de Física.
\end{abstract}

Palavras-chave: Acelerômetro, Atividades Experimentais, Laboratório de Física.

\begin{abstract}
This work investigated the feasibility of using the BBC micro: bit internal accelerometer in experimental activities. Two experiments were used for tests, which dealt with Newton's Second Law and the Rotation motion. In both, the objective was to directly evaluate the acceleration and thus analyze the data through descriptive statistics and graphical analysis, generating results that will serve as a reference for students in the laboratory, in addition to clarifying concepts of visualization and comprehension. The results obtained help to better understand what really happens in acceleration movements and can help to reorganize experimental activities for the teaching of Physics.
\end{abstract}

Keywords: Accelerometer, Experimental Activities, Physics Laboratory.

\section{Introdução}

O caráter experimental no ensino de Física está diretamente relacionado à importância do laboratório didático nos processos de ensino e aprendizagem. Esse ambiente propicia ao aluno a vivência e o manuseio de instrumentos, que possibilitarão o conhecimento de diversos tipos de atividades, as quais poderão estimular a curiosidade e a vontade em aprender e vivenciar a ciência, tal como ela é [1].

Porém, ao longo de várias décadas, a qualidade das atividades experimentais no ensino de Física em todos os níveis da educação tem sido objeto de reflexão. As atividades experimentais não podem ser vistas apenas como uma prática mecanizada, mas devem dar possibilidades de uma construção pessoal do conhecimento por parte dos alunos. Sendo assim, as tecnologias em geral podem ser importantes aliadas na renovação da função do laboratório de ensino se usadas de forma reflexiva e criteriosa. A inserção de Tecnologias da Informação e Comunicação (TIC) no ensino experimental pode auxiliar os estudantes a interagirem de forma consciente com os experimentos, propiciando melhores condições para a análise do que ocorre no mundo prático [2].

\footnotetext{
*Endereço de correspondência: diegodejesus15@hotmail.com
}

Existem inúmeros recursos tecnológicos cujo mercado internacional tem disponibilizado módulos sensores que, se apoiados numa eletrônica adequada, podem medir temperatura, campo magnético, deslocamento, pressão, aceleração, tempo, força, entre outras tantas grandezas físicas, que vêm se tornando acessíveis ao público em geral e que podem ser fortes aliados na construção de um novo cenário para o trabalho experimental no ensino de Física, principalmente na área de microcontroladores.

De baixo custo e dimensões limitadas, os microcontroladores possuem capacidade computacional limitada, porém seu uso se dá em diversas áreas, podendo ser utilizado na área de ensino. Nessa linha educacional, iniciativas que estimulem a utilização de microcontroladores em atividades de ensino têm surgido em diversos países, como o exemplo inglês denominado BBC micro: bit, ver Figura 1. Criado pela emissora britânica BBC juntamente com 29 empresas, o BBC micro: bit é um microcontrolador cuja principal ideia é ensinar programação para crianças de uma maneira simples, já que pode ser programado em linguagens como: Bocks, Javascript, Python e $\mathrm{C} / \mathrm{C}++$. Com 4 centímetros de largura por 5 centímetros de comprimento, possui uma porta microUSB, conexão Bluetooth, dois botões programáveis, um botão de reset, acelerômetro $3 \mathrm{D}$, magnetômetro $3 \mathrm{D}$ e 


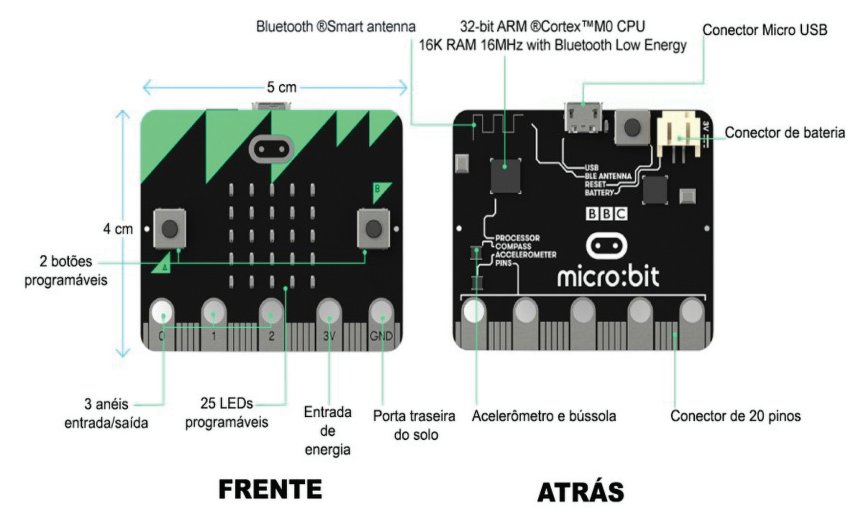

Figura 1: $\mathrm{BBC}$ micro: bit e seus componentes.

cinco entradas I/O (do inglês Input/Output) que podem ser conectadas a outros kits ou sensores externos [3].

Dentre os diversos atributos que o BBC micro: bit traz, pode-se destacar o seu acelerômetro, o qual será o foco de investigação deste trabalho.

\section{Acelerômetros}

A aceleração é a taxa com que a velocidade varia em relação ao tempo, podendo ser expressa matematicamente pela equação a seguir.

$$
\vec{a}=\frac{d \vec{v}}{d t}
$$

De modo geral um acelerômetro pode ser definido como um dispositivo que mede a vibração ou a aceleração estática/dinâmica do movimento de uma estrutura. Pode ser usado para medir inclinação, forças inerciais (velocidade, deslocamento ou força), choque ou vibração. Ao analisarmos a função do acelerômetro de medir a "aceleração própria", vemos que esse conceito é antigo e surgiu em 1915 pela Teoria da Relatividade Geral de Albert Einstein 4 .

Portanto, o acelerômetro não mede propriamente a aceleração de acordo com a equação (1), mas estima a grandeza

$$
\vec{a}^{\prime}=\vec{a}-\vec{A}
$$

onde $\vec{a}^{\prime}$ é a aceleração medida pelo acelerômetro, $\vec{a}$ é a aceleração medida por um referencial inercial e $\vec{A}$ é a aceleração do referencial não inercial medida por um referencial inercial [5].

Outro caso que será estudado neste trabalho é o acelerômetro em um movimento circular com velocidade angular constante. A aceleração medida pelo acelerômetro pode ser descrita pela equação (3).

$$
\vec{a}^{\prime}=\vec{a}-\vec{\omega} \times\left(\vec{\omega} \times \vec{r}^{\prime}\right)-2 \vec{\omega} \times \vec{v}^{\prime}-\frac{d \omega}{d t} \times \vec{r}^{\prime}
$$

Como podemos observar para uma partícula de massa $m$ observada a partir de um referencial não inercial,

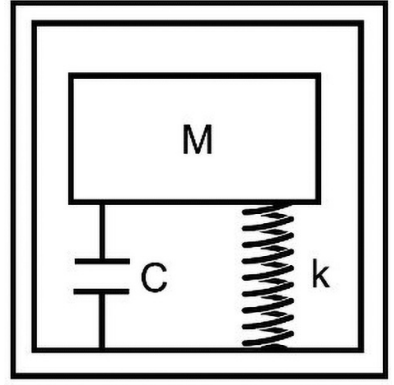

Figura 2: Representação conceitual de um acelerômetro.

podem aparecer até três forças fictícias: uma é $-m \vec{\omega} \times$ $\left(\vec{\omega} \times \vec{r}^{\prime}\right)$ que é conhecida como força centrífuga, porém como esse é um caso particular de movimento circular a expressão de força centrifuga pode ser escrita como $-\omega^{2} r^{\prime} \vec{r}^{\prime}$. A outra força fictícia, $-2 \vec{\omega} \times \vec{v}^{\prime}$, é chamada de força de Coriolis. Quanto à última força, $-\frac{d \omega}{d t} \times \vec{r}^{\prime}$, é nula, pois a velocidade angular é constante [5].

De forma geral os acelerômetros possuem como sistema básico de funcionamento um sistema massa-mola. As molas enquanto dentro da sua região linear são governadas pela Lei de Hooke, ver Figura 2

O corpo de massa $m$ é uma estrutura de silício e seu movimento é controlado por molas de polisilício. Quando o sistema é acelerado, de acordo com a Segunda Lei de Newton, existe uma força resultante igual ao produto da massa $(M)$ pela aceleração $(a)$. Essa força faz com que o corpo de massa expanda ou comprima o sistema de molas, resultando em uma força elástica, mais conhecida como Lei de Hooke. A Lei de Hooke diz que a força elástica é igual ao produto da constante elástica da mola $(k)$ pelo deslocamento da mesma $(x)$. Partindo dessa premissa pode-se chegar ao módulo da aceleração [4].

$$
\begin{aligned}
M a & =k x \\
a & =\frac{k}{M} x
\end{aligned}
$$

Logo, pode-se dizer que a aceleração medida é diretamente proporcional ao deslocamento. Para medir estes deslocamentos, são utilizadas placas paralelas de capacitores, porém cada capacitor é muito pequeno, ou seja, tem pouca capacitância e por essa razão são necessários diversos capacitores.

A capacitância de um capacitor paralelo é proporcional a permissividade do dielétrico $(\varepsilon)$ e a área da superfície das placas $(A)$, e inversamente proporcional a distância entre as placas $(x)$, conforme equação (6).

$$
C=\frac{\varepsilon A}{x}
$$

Portanto a distância é medida por

$$
x=\frac{\varepsilon A}{C}
$$




\section{Por que Usar o Acelerômetro do BBC Micro: Bit?}

O BBC micro: bit pode ser utilizado a partir das linguagens: Blocks, Javascript, MicroPython, $\mathrm{C} / \mathrm{C}++$, uLisp, TinyGo, etc. Ao nosso ver, a linguagem Python é a mais recomendada para atividades de coleta de dados em experimentos de laboratório didático, pois propiciam um vasto controle de parâmetros e uma extensa oferta de recursos.

O acelerômetro do BBC micro: bit é um Freescale MMA8653FC de 10 bits, portador da tecnologia MEMS 3D (Micro Eletrical-Mechanical System) 6], que é a essência da computação analógica, capaz de sentir e reagir a estímulos externos naturais, se aproximando muito mais da realidade, ao invés do comportamento binário e artificial da computação digital [7]. Vale ressaltar que a medição com o acelerômetro 3D do BBC micro: bit permite medir a aceleração diretamente, enquanto que em experimentos no laboratório, tal grandeza é medida a partir de equações da mecânica clássica, ou seja, de forma indireta, ignorando vários efeitos que influenciam a aceleração real, como vibrações do movimento e turbulência do ar.

Diversas técnicas e equipamentos são utilizados para medição da aceleração de um corpo. Acelerômetros piezoelétricos foram bastante utilizados nos últimos anos, os quais possuem um cristal como elemento sensor. Porém, atualmente existe uma gama de sensores disponíveis no mercado que medem aceleração, sendo que muitos desses são do tipo MEMS capacitivo, os quais são baseados em um sistema massa-mola e obedecem a Lei de Hooke. Os dispositivos possuem uma massa de silício e molas de polisilício. Ao se reduzir a constante elástica das molas, a precisão do dispositivo é aumentada, porém o torna menos resistente a choques [4]. Existem dispositivos de baixa aceleração, entre $9,7856 \mathrm{~m} / \mathrm{s}^{2}$ e $195,712 \mathrm{~m} / \mathrm{s}^{2}$, ou de alta aceleração, entre $195,712 \mathrm{~m} / \mathrm{s}^{2}$ e $978,56 \mathrm{~m} / \mathrm{s}^{2}$, com um, dois ou três eixos operacionais. Neste trabalho, o valor para aceleração da gravidade adotado foi de $9,7856 \mathrm{~m} / \mathrm{s}^{2}$, tendo como referência um estudo sobre as variações da aceleração gravitacional em função da altitude e latitude local [8].

\section{A Utilização do BBC Micro: Bit em Atividades Experimentais}

O BBC micro: bit foi utilizado em dois experimentos no laboratório de Física. O primeiro foi sobre a Segunda Lei de Newton, e o segundo experimento foi sobre Rotação. Para ambos foram a priori desenvolvidos os códigos fonte de programação, usando Micropython, que é uma linguagem Python otimizada para microcontroladores. Os códigos produzidos podem ser acessados em: https: //github.com/die-santos/Micro-Bit

Antes da utilização do acelerômetro nesses dois experimentos, foram realizadas medições do valor da gravidade local, utilizando diferentes escalas e frequências, com intuito de determinar qual a frequência e escala seriam mais apropriadas, estes valores são mostrados na Tabela 1

Foi utilizada a escala com frequência de $100 \mathrm{~Hz}$. As frequências mais altas geram maiores valores de incerteza e consequentemente diferentes valores da aceleração da gravidade. Além disso, frequências maiores gerariam muitos dados por segundo, os quais poderiam não ser devidamente armazenados no microcontrolador. Como havia conhecimento do resultado obtido para aceleração nos experimentos, calculado de forma indireta, foi utilizada a escala aceleração de $19,5712 \mathrm{~m} / \mathrm{s}^{2}$, pois essa era suficiente para as medições perante os valores das acelerações conhecidas.

No experimento sobre a Segunda Lei de Newton, o BBC micro: bit foi acoplado a um carrinho sobre um trilho de ar. O carrinho está conectado a uma massa por um fio, conforme a Figura 3. Um sistema magnético mantém o carrinho em repouso e o acionamento de uma chave faz com que o carrinho seja solto e entre em movimento, sendo acelerado pela massa na extremidade do fio. Antes do acionamento da chave, foi pressionado o botão "A" no BBC micro: bit, de modo que a coleta de dados sobre a aceleração possa ser inserida em um

Tabela 1: Medidas do valor da gravidade para diferentes escalas e frequências.

\begin{tabular}{|c|c|c|c|c|c|c|}
\hline \multirow{3}{*}{$\begin{array}{l}\text { Frequência } \\
(\mathrm{Hz})\end{array}$} & \multicolumn{2}{|r|}{$19,5712\left(\mathrm{~m} / \mathrm{s}^{2}\right)$} & \multicolumn{2}{|c|}{$39,1424\left(\mathrm{~m} / \mathrm{s}^{2}\right)$} & \multicolumn{2}{|c|}{$78,8248\left(\mathrm{~m} / \mathrm{s}^{2}\right)$} \\
\hline & & Desvio & & Desvio & & Desvio \\
\hline & Média & Padrão $+(0,5$ LSB $)$ & Média & Padrão + (1,0 LSB $)$ & Média & Padrão $+(2,0$ LSB $)$ \\
\hline 800 & 9,5263 & 0,1167 & 9,4999 & 0,1297 & 9,7690 & 0,1220 \\
\hline 400 & 9,5194 & 0,0706 & 9,4940 & 0,0650 & 9,7768 & 0,0404 \\
\hline 200 & 9,5126 & 0,0574 & 9,4881 & 0,0209 & 9,7856 & 0,0023 \\
\hline 100 & 9,4979 & 0,0283 & 9,4842 & 0,0003 & 9,7856 & 0,0023 \\
\hline 50 & 9,4920 & 0,0004 & 9,4842 & 0,0003 & 9,7856 & 0,0023 \\
\hline 12.5 & 9,4920 & 0,0004 & 9,4842 & 0,0003 & 9,7856 & 0,0023 \\
\hline 6.25 & 9,4920 & 0,0004 & 9,4842 & 0,0003 & 9,7856 & 0,0023 \\
\hline 1.56 & 9,4920 & 0,0004 & 9,4842 & 0,0003 & 9,7856 & 0,0023 \\
\hline
\end{tabular}




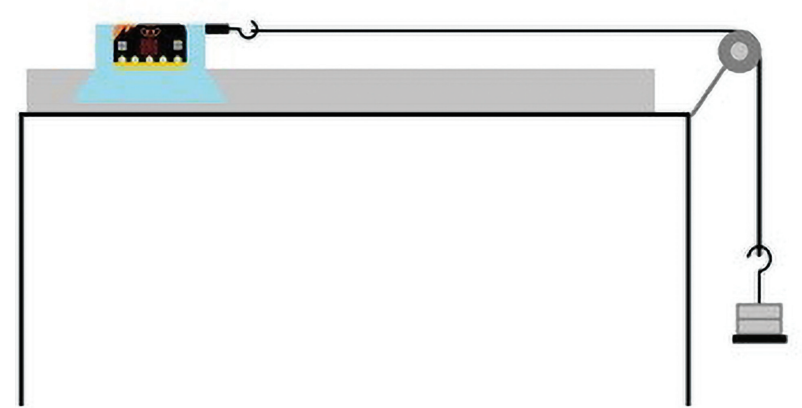

Figura 3: Esquema de montagem do experimento sobre a Segunda Lei de Newton com BBC micro: bit acoplado ao carrinho sobre o trilho de ar.

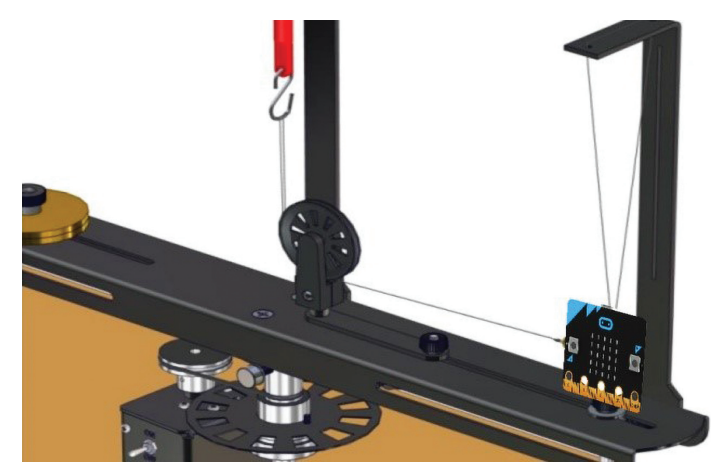

Figura 4: Esquema de montagem do experimento sobre Rotações com BBC micro: bit acoplado.

arquivo .csv, sendo que, via cabo micro USB, esses dados possam ser obtidos em tempo real utilizando o $\mathrm{Mu}$ Editor ${ }^{1}$ Assim, é possível fazer uma análise estatística e gerar gráficos da aceleração em função do tempo, sendo que para obter tais análises é necessário usar programas como Excel, LibreOffice Calc ou SciDavis. Ao todo foram feitas quatro medidas para a aceleração havendo uma permuta entre os corpos de prova que migraram do carrinho, com maior número de corpos de prova a priori, para o suporte.

No experimento sobre Rotação, ver Figura 4 o ideal é que o dispositivo já esteja em movimento para a coleta de dados. O micro: bit foi acoplando em uma base sobre um motor que pode realizar um movimento circular. Ao pressionar o botão "A", após 10 segundos é emitido um sinal sonoro e o micro: bit começa a registrar os dados em um arquivo .csv.

Ao contrário do movimento do experimento sobre a Segunda Lei de Newton, que mede a aceleração em apenas um eixo, no experimento de rotação a aceleração é medida em dois eixos, registrando assim duas tabelas

\footnotetext{
$1 \mathrm{Mu}$ é um editor de scripts Python com foco em programadores iniciantes. Escrito em Python e Qt5, facilita o processo de criação, execução e verificação de scripts Python. O Mu está disponível para instalação em: https://codewith.mu/en/download
}

de valores, uma para cada eixo. A análise de dados é feita da mesma forma que no experimento da Segunda Lei de Newton.

\section{Resultados e Discussões}

No experimento tradicional sobre a Segunda Lei de Newton, o objetivo é determinar o valor da aceleração do sistema a partir de medidas indiretas. Através da observação dos dados obtidos pelo micro: bit, os alunos podem obter uma medição direta para comparar e analisar seus resultados.

A análise gráfica permite uma obtenção de dados mais rápida e mais precisa. Na Figura 5 o intervalo inicial até a primeira linha tracejada em laranja mostra uma aceleração próxima de $0 \mathrm{~m} / \mathrm{s}^{2}$ indicando repouso do micro: bit, logo depois um pequeno pico indica um aumento na aceleração, ou seja, a partir desse ponto o micro: bit estava em movimento. Essa aceleração, teoricamente, deveria ser constante, mas pode se observar que os dados não apresentam uma linearidade e isso se deve às possíveis irregularidades no trilho de ar, a própria estabilidade do acelerômetro durante o movimento e aos furinhos do trilho de ar que geram turbulências, produzindo pequenas oscilações de aceleração. Verificouse que isso cresce com a intensidade do fluxo de ar do compressor. O ponto de colisão com o anteparo é apontado no gráfico. A medição com acelerômetro 3D do BBC micro: bit permitiu medir as acelerações reais do experimento e analisar fatores que eram até então desconsiderados.

O ideal é gerar estes gráficos para diferentes configurações alterando a massa do carrinho e do suporte na extremidade da linha, conforme as Figuras 6, 7 e 8 .

Para determinar a aceleração de forma indireta, se faz necessário estudar as forças que agem em cada corpo separadamente, e assim, compreender o sistema no todo. Para isso, diagramas de corpo livre são muito úteis e a partir desses é possível obter a aceleração para o sistema pela equação (8), na qual $P_{S}$ é o peso do suporte, $m_{C}$ é a massa do carrinho e $m_{S}$ a massa do suporte.

$$
a=\frac{P_{S}}{m_{C}+m_{S}}
$$

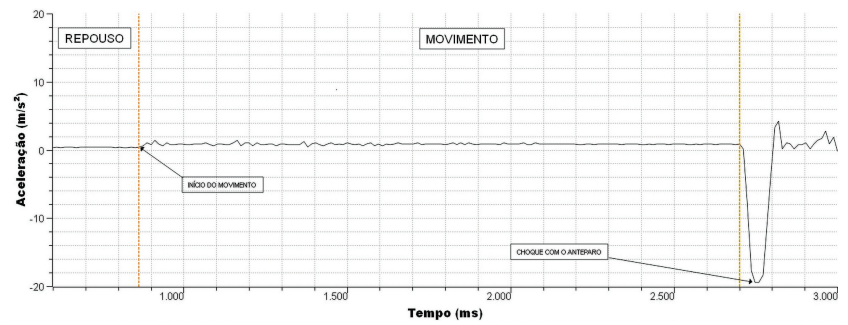

Figura 5: Aceleração em função do tempo para o micro: bit acoplado em um carrinho com massa total de $297,4 \mathrm{~g}$ e suporte com massa de $18,1 \mathrm{~g}$. 


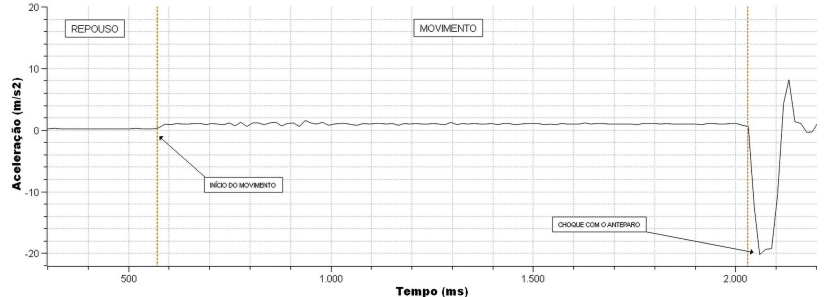

Figura 6: Aceleração em função do tempo para o micro: bit acoplado em um carrinho com massa total de $287,2 \mathrm{~g}$ e suporte com massa de $28,8 \mathrm{~g}$.

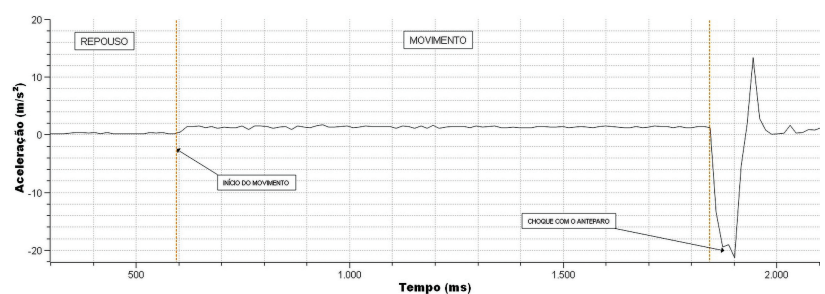

Figura 7: Aceleração em função do tempo para o micro: bit acoplado em um carrinho com massa total de $277,2 \mathrm{~g}$ e suporte com massa de $38,7 \mathrm{~g}$.

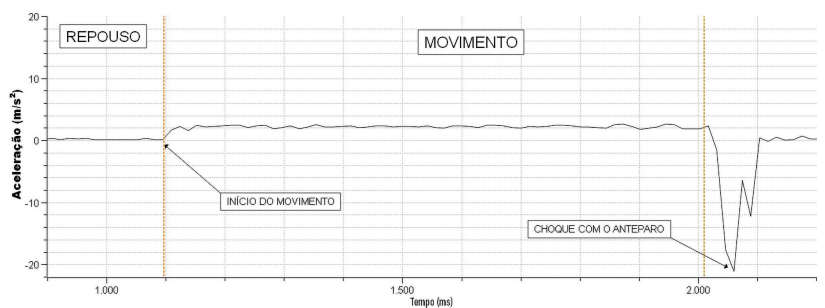

Figura 8: Aceleração em função do tempo para o micro: bit acoplado em um carrinho com massa total de $247,0 \mathrm{~g}$ e suporte com massa de $69,3 \mathrm{~g}$.

A incerteza da aceleração é dada pela equação (9), onde $\sigma_{P_{S}}, \sigma_{m_{S}}$ e $\sigma_{m_{C}}$, são as incertezas do peso do suporte, da massa do suporte e da massa do carrinho, respectivamente.

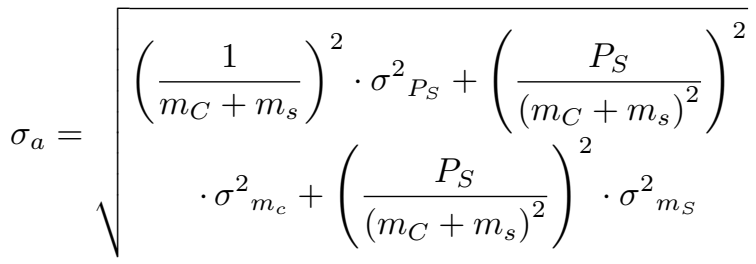

A Tabela 2 contém os valores encontrados para a aceleração usando as equações (8) e (9) e os valores obtido via BBC micro: bit.

A Tabela 2 mostra diferença nos valores de aceleração, principalmente para pequenos valores de massa do suporte. Isso ocorre porque a força Peso referente ao suporte é a responsável pelo movimento do carrinho, ou seja, quanto maior for a massa do suporte melhor será a aproximação entre os dados coletados pelo BBC micro: bit e os valores encontrados a partir da equação (8).

Comparando os gráficos anteriores e os dados da Tabela 2 é nítida a relação entre as variáveis da Segunda Lei de Newton, pois a cada momento que se diminui a massa do carrinho e aumenta a massa do suporte, a aceleração também aumenta.

No experimento de Rotação, uma partícula está em movimento circular uniforme quando descreve uma circunferência ou um arco de circunferência com velocidade escalar constante. Embora a velocidade escalar não varie, o movimento é acelerado porque a velocidade muda de direção.

A velocidade está sempre na direção tangente à circunferência e tem o mesmo sentido do movimento, enquanto a aceleração está sempre na direção radial e aponta para o centro do círculo. Por essa razão, a aceleração associada ao movimento circular uniforme é chamada de aceleração centrípeta, onde o módulo dessa aceleração é obtido a partir da equação seguinte [9].

$$
a_{c p}=\frac{v^{2}}{R}
$$

Porém, conforme foi discutido na seção 2, o BBC micro: bit é um referencial não inercial, logo o que é medido pelo acelerômetro não é a aceleração centrípeta, mas a aceleração centrífuga. A aceleração centrífuga possui o mesmo módulo e direção que a aceleração centrípeta, apenas o sentido que se opõe, apontando para fora do centro da circunferência. Diante disso, foi necessário acrescentar uma pequena mudança no código fonte onde o valor da aceleração medido corresponde ao módulo da aceleração centrífuga.

A posição do micro: bit foi ajustada para que o raio da trajetória fosse de 0,1630 $\pm 0.0005 \mathrm{~m}$. A massa do micro: bit foi mensurada no valor de $0,0571 \pm 0,0011 \mathrm{~kg}$ e logo em seguida foi pendurado de maneira que o fio fique na vertical, coincidindo com a abertura do suporte. Segurando o micro: bit nessa posição o dinamômetro foi ajustado para medir uma força de $0,2 \pm 0,1 \mathrm{~N}$.

Tabela 2: Valores da aceleração obtidos de forma Direta e Indireta.

\begin{tabular}{lccc}
\hline Massa do Carrinho $(\mathrm{kg})$ & Massa do Suporte $(\mathrm{kg})$ & Aceleração Direta $\left(\mathrm{m} / \mathrm{s}^{2}\right)$ & Aceleração Indireta $\left(\mathrm{m} / \mathrm{s}^{2}\right)$ \\
\hline $0,2974 \pm 0,0060$ & $0,0181 \pm 0,0004$ & $0,5608 \pm 0,0158$ & $0,7544 \pm 0,0979$ \\
$0,2872 \pm 0,0057$ & $0,0288 \pm 0,0006$ & $0,8909 \pm 0,0233$ & $0,9858 \pm 0,1183$ \\
$0,2772 \pm 0,0055$ & $0,0387 \pm 0,0007$ & $1,1975 \pm 0,0282$ & $1,2994 \pm 0,0899$ \\
$0,2470 \pm 0,0049$ & $0,0693 \pm 0,0014$ & $2,1417 \pm 0,0474$ & $2,1737 \pm 0,1945$ \\
\hline
\end{tabular}


Ao ligar fonte de tensão a plataforma começa a rotacionar e a tensão foi ajustada de forma que o dinamômetro volte a indicar a força ajustada inicialmente. $\mathrm{O}$ sistema foi deixado girando por certo tempo para que ele se estabilizasse. Com um cronômetro manual foi medido o período para 20 rotações. O mesmo procedimento foi feito para forças iguais a $0,4 \pm 0,1 \mathrm{~N}$ e $0,6 \pm 0,1 \mathrm{~N}$, respectivamente.

Com estes dados foram obtidos valores para a aceleração teórica via equação 11 e a incerteza associada é dada pela equação 12

$$
\begin{aligned}
a_{c p} & =\frac{F_{c p}}{m} \\
\sigma_{a_{c p}} & =\sqrt{\left(\frac{1}{m}\right)^{2} \sigma_{F_{c p}}{ }^{2}+\left(\frac{F_{c p}}{m^{2}}\right)^{2} \sigma_{m}^{2}}
\end{aligned}
$$

onde $F_{c p}$ é a força centrípeta, $m$ é a massa, $\sigma_{a_{c p}}$ é a incerteza associada a aceleração, $\sigma_{F_{c p}}$ é a incerteza associada a força centrípeta e $\sigma_{m}$ é a incerteza associada a massa.

Os valores encontrados para a aceleração teórica e a aceleração medida pelo micro: bit estão na Tabela 3

A análise da Tabela 3 mostra que os valores para a aceleração teórica e o medido com o micro: bit são bem próximos. Além disso, é possível notar uma relação direta entre a aceleração centrípeta e a força centrípeta, que está de acordo com a Segunda Lei de Newton.

A partir do uso de equações para o movimento circular é possível obter outros parâmetros, como a velocidade angular e linear. Outra análise possível é a partir do gráfico de Aceleração Centrípeta x Tempo, conforme a Figura 9

A aceleração centrípeta é uma grandeza que se mantém constante ao longo do movimento circular, porém devido a instabilidade que a base do experimento tem

Tabela 3: Valores da aceleração obtidos de forma Indireta e Direta.

\begin{tabular}{ccc}
\hline & $\begin{array}{c}\text { Aceleração } \\
\text { Indireta }\left(\mathrm{m} / \mathrm{s}^{2}\right)\end{array}$ & $\begin{array}{c}\text { Aceleração } \\
\text { Direta }\left(\mathrm{m} / \mathrm{s}^{2}\right)\end{array}$ \\
\hline $0,2000 \pm 0,1000$ & $3,5026 \pm 1,7526$ & $3,7070 \pm 0,1650$ \\
$0,4000 \pm 0,1000$ & $7,0053 \pm 1,7565$ & $7,3580 \pm 0,0940$ \\
$0,6000 \pm 0,1000$ & $10,5079 \pm 1,7630$ & $10,4290 \pm 0,1220$ \\
\hline
\end{tabular}

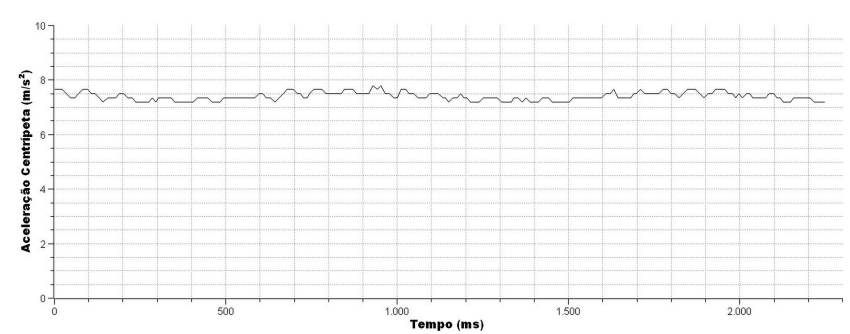

Figura 9: Aceleração Centrípeta em função do tempo.

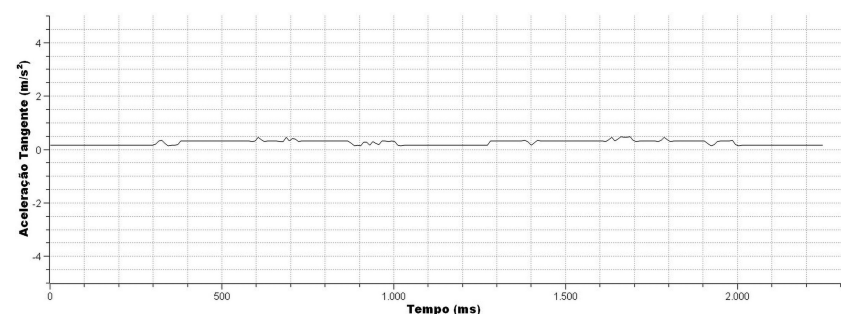

Figura 10: Aceleração Tangente em função do tempo.

devido ao movimento de rotação, vemos pequenas oscilações no gráfico da Figura 9 .

Outro gráfico que pode ser gerado a partir dos dados coletados é a relação entre a aceleração tangente e o tempo, mostrado na Figura 10.

Conforme esperado, a aceleração tangente tem um valor próximo a $0 \mathrm{~m} / \mathrm{s}^{2}$, sendo o valor médio igual a $0,2418 \pm 0,0888 \mathrm{~m} / \mathrm{s}^{2}$. Estes dados fortalecem a ideia de que mesmo que haja uma aceleração no movimento circular, a velocidade linear é constante.

\section{Considerações Finais}

As atividades em laboratório são centradas na coleta de dados e com pouco ou nenhum tempo dedicado à discussão dos conceitos e princípios físicos envolvidos. Isso não apenas diminui a eficácia pedagógica desses laboratórios; o tempo excessivo gasto em ações repetitivas e pouco inspiradoras como a coleta e sistematização dos dados contribui para que boa parte dos alunos perca o interesse pelas atividades práticas, terminando por considerá-las uma perda de tempo 10 .

Os resultados apresentados neste trabalho mostram que o micro: bit possui capacidade pra ser usado em experimentos onde exista medida de aceleração e os dados podem ser apresentados com rapidez e uma certa precisão, abrindo espaço para discussões, voltadas principalmente para o modelo Físico estudado em sala de aula e a realidade vista em laboratório. É interessante salientar que a aceleração raramente é medida de forma direta em laboratórios, pois acelerômetros costumam ser caros e difíceis de operar.

Todo o ferramental de programação se mostrou de caráter prático e bem fácil, pois existe um bom material disponível para consulta e são necessárias poucas linhas de códigos fontes para se obter o resultado desejado. Para experimentos mais complexos, espera-se que as incertezas sejam maiores, porém, isso não impediria a utilização desse sensor se considerarmos que as atividades de ensino não exigem grandes precisões, além da frequência e escala usadas para medir a aceleração poderem ser alteradas no código fonte, o que permite melhorar os dados e consequentemente os resultados.

É claro que o micro: bit não se limita aos experimentos apresentados. Existe um leque de atividades que envolvem a medida da aceleração e outros mais que 
podem ser feitos utilizando os demais sensores que ele possui.

Com este trabalho, espera-se fomentar a reorganização de roteiros e atividades experimentais no laboratório de Física fazendo com que a reflexão dê lugar a repetição, além de propiciar a implementação de novas atividades experimentais, as quais atualmente são inviáveis devido à falta de equipamentos de baixo custo para serem utilizados nas medições de aceleração. Pode-se concluir que esses sensores têm potencial para atividades de ensino e podem contribuir para aulas de ciências, especialmente as de Física.

\section{Referências}

[1] N.A. Grandini e C.R. Grandini, in Anais do XI Encontro de Pesquisa em Ensino de Física, editado por N.M.D. Garcia (Sociedade Brasileira de Física, São Paulo, 2010), p. 269.

[2] P.F.T. Dornelles, I.S. Araújo e E.A. Veit, Ciência \& Educação (Bauru) 18, 99 (2012).

[3] The Micro: bit Foudation: BBC micro: bit MicroPython documentation, disponível em: http://microbit-mic ropython.readthedocs.io/en/latest/\#, acessado em 20/11/2017.

[4] S.M. Massuda, Uso de acelerômetro MEMS para aferir o desempenho de automóveis. Trabalho de Conclusão de Curso, Centro Universitário de Brasília, Brasília (2007).

[5] J.B. Neto, Mecânica Newtoniana, Lagrangiana e Hamiltoniana (Editora Livraria da Física, São Paulo, 2004) p. 98.

[6] Acelerômetro MMA8653FC, disponível em: https://ww w.nxp.com/products/sensors/accelerometers/2g-4g8g-low-g-10-bit-digital-accelerometer:MMA8653FC, acessado em 18/09/2021.

[7] P. Saffo, Communications of the ACM 40, 93 (1997).

[8] W. Lopes, Caderno Brasileiro de Ensino de Física 25, 561 (2008).

[9] D. Halliday e R. Resnick, Fundamentos da Física: Mecânica (LTC, Rio de Janeiro, 2008), v. 1, p. 77.

[10] L.P. Vieira e C.E. Aguiar, Experimentos com o acelerômetro de Tablets e Smartphones. Dissertação de Mestrado, Universidade Federal do Rio de Janeiro, Rio de Janeiro (2013). 